CLINICAL STUDY

\title{
Weight loss larger than $10 \%$ is needed for general improvement of levels of circulating adiponectin and markers of inflammation in obese subjects: a 3-year weight loss study
}

\author{
Erik L Madsen, Aila Rissanen ${ }^{2}$, Jens M Bruun, Kristin Skogstrand ${ }^{3}$, Serena Tonstad ${ }^{1}$, David M Hougaard ${ }^{3}$
} and Bjørn Richelsen

Department of Endocrinology and Metabolism C, Aarhus University Hospital, Aarhus Sygehus, Tage Hansensgade 2, DK-8000 Aarhus C, Denmark, ${ }^{1}$ Department of Preventive Cardiology, Ulleval Hospital, SFFM N-0407 Oslo, Norway, ${ }^{2}$ Obesity Research Unit, Helsinki University Hospital, 00260 Helsinki, Finland and ${ }^{3}$ Department of Clinical Biochemistry, Statens Serum Institut, Artillerivej S, DK-300 Copenhagen, Denmark

(Correspondence should be addressed to B Richelsen; Email: ovl06br@as.aaa.dk)

\begin{abstract}
Objective: To investigate the effects of: I) short- ( 8 weeks), II) long-term (3 years) weight loss, and III) the degree of weight loss on circulating levels of adiponectin, high sensitive-C reactive protein (hs-CRP), and fibrinogen in obese subjects. Moreover, to evaluate the effect of the lipase inhibitor, orlistat, on these parameters.

Design: Weight loss induced in 93 obese subjects (mean weight: $108.9 \pm 15.8 \mathrm{~kg}$ ) through 8 -week very-low-energy diet (VLED, $800 \mathrm{kcal} /$ day) followed by randomization to orlistat or placebo together with lifestyle intervention for further 3 years. Adiponectin and hs-CRP were measured at baseline, after 8 weeks of VLED and 6, 12, and 36 months after the VLED by flowmetric xMAP technology (Luminex Multi-Analyte Profiling System, Luminex Corp., Austin, TX, USA). Fibrinogen was measured in a coagulation assay.

Results: Weight loss after VLED treatment was $14.3 \pm 4.5 \mathrm{~kg}$ and after 3 years $7.7 \pm 8.7 \mathrm{~kg}$. Orlistattreated subjects regained $3.9 \mathrm{~kg}$ less than placebo-treated from the end of the VLED to 3 years $(P=0.01)$. No differences were detected between the two groups regarding changes in adiponectin, hs-CRP, or fibrinogen. Accordingly, the groups were combined for further analyses. Serum adiponectin increased by $22 \%(P<0.05)$ after the VLED but returned to baseline after 3 years. Both short- and long-term weight losses needed to be in excess of $10 \%(\sim 12 \mathrm{~kg})$ in order to increase adiponectin levels significantly. Weight loss was associated with a significant decrease in hs-CRP. Fibrinogen decreased by $12 \%(P<0.05)$ after 3 years.

Conclusions: In obese subjects, weight loss was associated with an increase in serum adiponectin and a decrease in hs-CRP and plasma fibrinogen. Long-term weight loss ( 3 years) must exceed $10 \%$ to induce a combined significant improvement in these inflammatory markers.
\end{abstract}

European Journal of Endocrinology 158 179-187

\section{Introduction}

Obesity is associated with a propensity towards the development of dyslipidemia, insulin resistance, hyperglycemia, hypertension, and a state of chronic lowgrade inflammation, leading to an increased risk of cardiovascular morbidity and mortality (1-4). Human adipose tissue (AT) is not only a storage organ for excess energy but also an active endocrine organ known to produce and release a number of bioactive proteins known as adipokines, e.g., adiponectin, leptin, tumor necrosis factor (TNF)- $\alpha$, interleukin (IL)-6, IL-8, and monocyte chemoattractant protein (MCP)-1, suggested to exert paracrine or endocrine effects (5-7). The obesity-associated low-grade inflammation may be caused by an increase in pro-inflammatory adipokines such as IL-6, TNF- $\alpha$, IL- 8 , and MCP-1 and a decrease in the anti-inflammatory adipokine adiponectin, contributing to the development of long-term complications such as insulin resistance, endothelial dysfunction, and atherosclerosis (8-10). From animal models, it is found that adiponectin may improve insulin sensitivity as well as decrease inflammation and atherogenesis (11-13). Plasma adiponectin has an inverse correlation with measures of body fat (4) and insulin sensitivity (13). Weight loss is associated with an increase in plasma adiponectin in some (14-16) but not all studies $(17,18)$. It remains unknown whether these opposing findings are due to differences in the degree of weight loss or the way weight loss is achieved, for example, short- versus long-term weight loss. Thus, the aim of the present study was to investigate the effect of both 
short- (8 weeks of very-low-energy diet, VLED) and long-term weight loss ( 3 years) on plasma levels of antiand pro-inflammatory markers (e.g., adiponectin, hs-CRP, fibrinogen) in abdominally obese subjects and to assess the level of weight loss needed to achieve a long-term improvement in these markers. Moreover, we investigated whether the anti-obesity drug, orlistat (a lipase inhibitor), had an independent effect on these parameters in a randomized controlled study.

\section{Materials and methods}

\section{Study design}

The present study is a sub-study of the Scandinavian multicenter study of obese subjects with the metabolic syndrome (SMOMS) which was performed to investigate whether treatment with the lipase inhibitor, orlistat, led to greater weight loss in the long term (3 years) as compared with placebo in a randomized placebocontrolled study (19). The present study consists of a subgroup of 93 subjects ( 49 in the orlistat group and 44 in the placebo group) who completed all visits of the SMOMS trial. Inclusion criteria were age between 18 and 65 years with a body mass index (BMI) of $\geq 30-\leq 45 \mathrm{~kg} / \mathrm{m}^{2}$ and a waist circumference $\geq 102$ (male) or $\geq 92 \mathrm{~cm}$ (female) with at least one of the following risk factors: impaired fasting glucose $(\geq 6.1 \mathrm{mmol} / \mathrm{l})$, manifest type 2 diabetes (only diet treated), or dyslipidemia (high density lipoprotein (HDL) cholesterol $\leq 0.9$ (male) or $\leq 1.1 \mathrm{mmol} / \mathrm{l}$ (female) and/or serum triglycerides $\geq 2.0-<10 \mathrm{mmol} / \mathrm{l}$ ). Exclusion criteria were drug-treated diabetes mellitus, severe dyslipidemia (serum triglycerides $\geq 10 \mathrm{mmol} / \mathrm{l}$ ), use of lipid-lowering agents, uncontrolled hypertension, or significant gastrointestinal problems. The $5 \%$ weight loss criterion during the VLED for continuation into the randomized treatment phase was fulfilled by $81 \%$ of the whole study population and $65 \%$ of the randomized subjects completed 36 months of treatment in the trial with no significant differences in dropout rates between the treatment groups (19). The study was conducted in accordance with the Declaration of Helsinki and was approved by the Ethical Committees in Denmark, Norway, and Finland. Written informed consent was obtained from all participants.

\section{Lifestyle interventions and treatment}

All subjects were initially asked to complete 8 weeks of VLED (600-800 kcal/day; Modifast (Novartis, Basel, Switzerland) or Nutrilett (Nycomed Pharma, Oslo, Norway)). Those subjects who were able to lose at least $5 \%$ of their initial body weight during the VLED phase were allowed to continue into the controlled doubleblinded treatment phase of the study and were randomly assigned to either orlistat (120 mg three times daily) or matching placebo capsules for further 3 years. Both treatment groups were supplemented with the same dietary and exercise counseling (19). In brief, the subjects were seen by dieticians ten times during the treatment period and advised to follow a diet with a reduced fat energy content in order to avoid weight regain and to avoid side effects of the lipase inhibitor. Energy intake and compliance to the diet were formally assessed at baseline and three times from after the VLED to 3 years. Average daily intake of total calories as well as fat energy content and cholesterol was significantly reduced from baseline to 3 years $(P<0.05$; data not shown). The subjects were encouraged to increase their level of physical activity, and their physical fitness was assessed with the Urho Kaleva Kekkonen (UKK) institute walking test (20). The physical fitness increased significantly $(P<0.05)$ with no differences between the treatment groups in the original study (data not shown).

Blood sampling and anthropometrical measurements were done before and after the VLED period - and after 6 months, 1 , and 3 years of treatment. The time course of the variables was evaluated over three periods: period I, from baseline to the end of the VLED; period II, from the end of the VLED to 3 years; and period III, from baseline to 3 years (see Table 1 ).

\section{Measurements}

Body weight, waist, and hip circumference were measured by standardized techniques. Plasma samples from all time points were analyzed together for fibrinogen (Clauss coagulation assay (21)), lipids, glucose, and insulin using standardized assays (NOVA Medical, Medilab A/S, Copenhagen, Denmark). Adiponectin and hs-CRP were analyzed together in serum by three-plexed sandwich immunoassays based on flowmetric xMAP technology (Luminex multi-analyte profiling system, Luminex Corp., Austin, TX, USA) performed and characterized essentially as described (22). In short, the serum samples were diluted 1:100 in buffer and set up in filter plate wells for duplicate determination. To each filter plate well we added $50 \mu \mathrm{l}$ diluted sample and $50 \mu \mathrm{l}$ suspension of capture antibody-conjugated beads, and 1500 beads per analyte. After $1 \frac{1}{2} \mathrm{~h}$ of incubation, the beads were washed twice and subsequently reacted for $1 \frac{1}{2} \mathrm{~h}$ with a mixture $(50 \mu \mathrm{l})$ of relevant biotinylated detection antibodies, each diluted $1: 1000$, before $50 \mu \mathrm{l}$ streptavidin-phycoerythrin, $20 \mu \mathrm{g} / \mathrm{ml}$ was added to the wells, and the incubation was continued for an additional $30 \mathrm{~min}$. The beads were finally washed twice and resuspended in $125 \mu \mathrm{l}$ buffer and analyzed on the Luminex 100 platform according to manufacturer's instructions. The intra- and inter-assay coefficients of variation $(\mathrm{CV})$, assessed by repeated measurements on normal control plasma samples, for adiponectin were 4 and $23 \%$ respectively and for hs-CRP were 12 and $13 \%$ respectively. The working range for each analyte was 
Table 1 Anthropometrical data, inflammatory markers, and lipids according to treatment group.

\begin{tabular}{|c|c|c|c|c|}
\hline \multirow[b]{3}{*}{ Variable } & \multirow[b]{3}{*}{ Treatment } & \multicolumn{3}{|c|}{ Period III } \\
\hline & & \multicolumn{2}{|l|}{ Period I } & Period II \\
\hline & & Baseline & After 8 weeks of VLED & 3 years \\
\hline \multirow[t]{2}{*}{ Weight (kg) } & Orlistat & $109(104.5-113.8)$ & $95.8(91.8-100.1)$ & $99.9(95-105.1)^{\star}$ \\
\hline & Placebo & $109.9(105.1-115)$ & $95.6(91.3-100.1)$ & $105(99.4-110.9)$ \\
\hline \multirow[t]{2}{*}{ BMI $\left(\mathrm{kg} / \mathrm{m}^{2}\right)$} & Orlistat & $37.2(35.9-38.5)$ & $32.7(31.5-33.9)$ & $33.9(32.4-35.4)^{\star}$ \\
\hline & Placebo & $37.7(36.4-39.1)$ & $32.8(31.6-34.1)$ & $35.8(34.1-37.6)$ \\
\hline \multirow{2}{*}{ Waist circumference $(\mathrm{cm})$} & Orlistat & $117.4(114-121)$ & 106.5 (103.3-109.9) & $108.7(104.7-112.8)^{*}$ \\
\hline & Placebo & $117.7(114-121.6)$ & $105.9(102.4-109.4)$ & $112.2(107.7-116.8)$ \\
\hline \multirow{2}{*}{ Adiponectin $(\mu \mathrm{g} / \mathrm{ml})$} & Orlistat & $0.38(0.32-0.45)$ & $0.49(0.41-0.58)$ & $0.43(0.35-0.53)$ \\
\hline & Placebo & $0.44(0.36-0.53)$ & $0.5(0.42-0.6)$ & $0.42(0.33-0.52)$ \\
\hline \multirow[t]{2}{*}{ Hs-CRP ( $\mu \mathrm{g} / \mathrm{ml})$} & Orlistat & $2.5(2.3-2.6)$ & $2.6(2.3-2.8)^{\dagger}$ & $2.4(2.2-2.5)$ \\
\hline & Placebo & $2.3(2.2-2.5)$ & $2.2(2-2.4)$ & $2.2(2-2.4)$ \\
\hline \multirow[t]{2}{*}{ Fibrinogen (g/l) } & Orlistat & $4.3(3.9-4.7)$ & $4.9(4.6-5.2)$ & $3.9(3.7-4.1)$ \\
\hline & Placebo & $4.4(4-4.8)$ & $4.8(4.5-5.1)$ & $3.6(3.4-3.8)$ \\
\hline \multirow[t]{2}{*}{ Total cholesterol $(\mathrm{mmol} / \mathrm{l})$} & Orlistat & $6(5.5-6.4)$ & $4.9(4.5-5.3)$ & $5.6(5.2-5.9)$ \\
\hline & Placebo & $5.8(5.4-6.3)$ & $4.5(4.2-4.9)$ & $5.4(5.1-5.8)$ \\
\hline \multirow[t]{2}{*}{ HDL cholesterol (mmol/l) } & Orlistat & $1.15(1.07-1.23)$ & 1.09 (1.01-1.17) & $1.14(1.05-1.24)$ \\
\hline & Placebo & $1.16(1.08-1.25)$ & $1.08(1-1.17)$ & $1.17(1.06-1.28)$ \\
\hline \multirow[t]{2}{*}{ LDL cholesterol (mmol/l) } & Orlistat & $3.8(3.4-4.1)$ & $3(2.8-3.4)$ & $3.4(3.1-3.8)$ \\
\hline & Placebo & $3.5(3.2-3.9)$ & $2.7(2.4-3)$ & $3.2(2.9-3.5)$ \\
\hline \multirow[t]{2}{*}{ Triglyceride $(\mathrm{mmol} / \mathrm{l})$} & Orlistat & $2(1.7-2.3)$ & $1.5(1.2-1.7)$ & $1.8(1.5-2.1)$ \\
\hline & Placebo & $2.2(1.8-2.6)$ & $1.5(1.3-1.8)$ & $1.9(1.6-2.3)$ \\
\hline
\end{tabular}

The patients were randomized to orlistat and placebo after 8 weeks of VLED. ${ }^{\star} P<0.05$ : differences between changes from the end of the VLED to 3 years (period II) according to treatment groups. ${ }^{\dagger} P<0.05$ : differences between levels of treatment groups at noted time point. Values are geometric means (except hs-CRP: arithmetic mean) with $95 \%$ confidence intervals adjusted for gender, age, site, and baseline fasting plasma glucose category (normal versus elevated) $n=66-68$.

assessed from the precision profile and defined as the concentration range where the CV is below $20 \%$. The range of concentrations investigated and approved for adiponectin was $1.95-1100 \mathrm{ng} / \mathrm{ml}$ and for hs-CRP was $0.2-5.12 \mu \mathrm{g} / \mathrm{ml}$. As compared with conventional ELISA measurement, the level of adiponectin was lower when measured by the present Luminex method. In evaluation studies, we found by measuring random blood samples from the present study that the mean value of adiponectin was $0.77 \pm 0.45 \mu \mathrm{g} / \mathrm{ml}$ measured by Luminex and $3.20 \pm 1.49 \mu \mathrm{g} / \mathrm{ml}$ by ELISA (B-Bridge International, San Jose, CA, USA). Thus, the present adiponectin levels should be added with 1.77 and multiplied by 1.84 as compared with the levels obtained by ELISA. This may be due to the interference of heterophile antibodies which are well-recognized causes of interference in immunoassays (23). The heterophile antibodies can cause false-negative results if binding directly to the capture antibodies and thereby blocking the reactive site from binding the specific analyte (adiponectin). Homeostasis model assessment of insulin resistance (HOMA-IR) was calculated as HOMA-IR = (fasting p-glucose $\times$ fasting p-insulin)/22.5.

\section{Statistical analysis}

The dropout rates for the involved sites did not differ significantly by the treatment groups or gender. Data were checked for normal distribution and transformed by logarithm base 'e' if needed. Absolute values (log base e) apart from hs-CRP which conformed better to normal distribution in untransformed values and changes were analyzed in a linear mixed model with unstructured covariance structure adjusting for gender, site, age, treatment, and baseline fasting plasma glucose category (normal versus elevated glucose $\geq 6.1 \mathrm{mmol} / \mathrm{l}$ ) together with interaction for time. For analysis of log base e changes during periods I-III, a multiple linear regression analysis was used including adjustment for gender, age, site, baseline fasting plasma glucose category, and changes in weight. For analysis of relative changes of non-transformed data, values were indexed with each individual's baseline value set as $100 \%$, and nonparametric statistics were applied: Friedman repeated measurements ANOVA on ranks with modification for multiple comparisons against control and the MannWhitney test were used when appropriate. Correlations were analyzed by the Spearman rho method for nonnormally and by the Pearson method for normally distributed parameters. The statistical software used for the analysis was Sigma-Stat v. 3.1, Systat Software, Inc. and SPSS v. 14.0, SPSS, Chicago, IL, USA). Results are geometric means with $95 \%$ confidence intervals or medians (25th-75th percentiles) unless otherwise noted. The number of participants was 93, but for adiponectin and hs-CRP only samples from 68 subjects were eligible for analysis. Thus, the exact numbers of participants included in the various measurements are stated in the figures and tables. A $P$ value $<0.05$ was considered statistically significant. 


\section{Results}

\section{Baseline characteristics}

Baseline characteristics are given in Table 1. The participants were obese with a mean BMI of $37 \mathrm{~kg} / \mathrm{m}^{2}$ and had elevated total cholesterol $(6.0 \pm 1.2 \mathrm{mmol} / \mathrm{l})$, glucose $(6.6 \pm 1.6 \mathrm{mmol} / \mathrm{l})$, insulin $(15.2 \pm 8.1 \mathrm{IU} / \mathrm{l})$, and systolic and diastolic blood pressure (150/ $96 \mathrm{mmHg}$ ). Mean age was $45.8 \pm 8.2$ years, and the proportion of males, was $54.4 \%$. The two treatment groups did not differ significantly concerning age or proportion of males, anthropometrical variables or lipids (Table 1) nor concerning blood pressure (148/94 vs $151 / 97 \mathrm{mmHg})$ or HOMA-IR $(4.2 \pm 3.1$ vs $5.1 \pm 3.7)$.

\section{Weight loss}

VLED treatment for 8 weeks (period I) induced a mean weight loss of $14.3 \pm 4.5 \mathrm{~kg}$ corresponding to $13.1 \%$ of initial body weight (Fig. 1). From the end of the VLED to the end of the 3-year trial (period II), the total weight regain was significantly smaller in the orlistat group compared with the placebo group $(4.8 \pm 8.5$ vs $8.7 \pm$ $6.3 \mathrm{~kg}, P=0.01$; Table 1 showing adjusted values; Fig. 1). However, when calculating the total weight loss from baseline to 3 years (period III), the weight loss was $2.6 \mathrm{~kg}(8.9 \pm 8.3 \mathrm{vs} 6.3 \pm 9.1 \mathrm{~kg})$ larger in the orlistat group compared with the placebo group. This difference did not, however, reach statistical significance $(P=0.16$; for unadjusted values). The orlistat group continued losing weight up to 6 months after the VLED $(P<0.05$; Fig. 1), whereas the placebo group regained weight at all visits following the VLED. Also, regain of BMI and waist circumference from the end of the VLED to the end of the 3-year trial (period II) was significantly

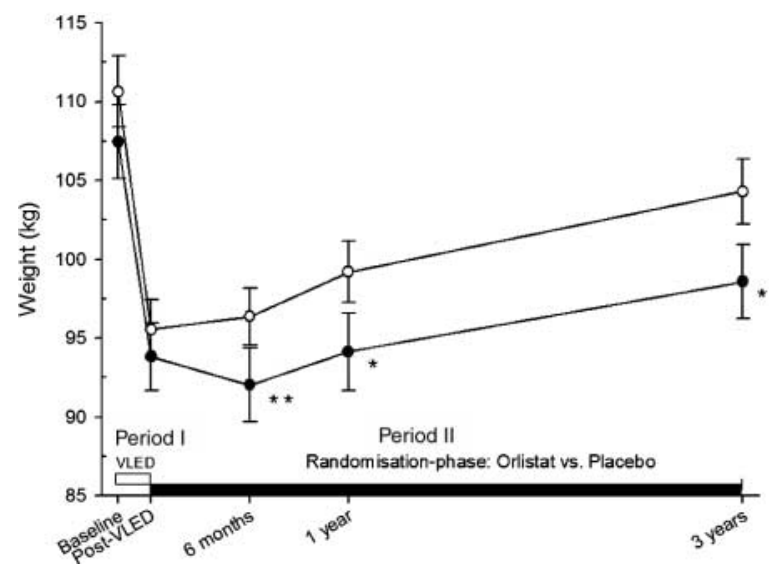

Figure 1 Weight changes during the study. After 8 weeks of VLED, the patients were randomized to either orlistat (๑) or placebo (o) for a further 3 years. Data are mean \pm S.E.M. $N=93$. ${ }^{\star} P<0.05$ and ${ }^{* *} P<0.01$ for differences in weight regain between treatment groups from the end of the VLED. reduced in the orlistat group $(P<0.05)$ compared with placebo (Table 1). Regarding the overall outcome of weight changes from baseline to 3 years (period III) in the two groups together: $31 \%$ of the subjects lost more than $10 \%$ of their initial body weight, $29 \%$ of the subjects lost between 5 and $10 \%$, another 29\% lost from 0 to $5 \%$, and $11 \%$ of the subjects gained weight compared with baseline levels. These proportions did not differ significantly $\left(P=0.29, \chi^{2}\right)$ between the two treatment groups (data not shown).

\section{Effect of treatment and weight changes on adiponectin, hs-CRP and fibrinogen}

Changes in adiponectin, hs-CRP and fibrinogen did not differ significantly between the treatment groups during period I (baseline to after the VLED), period II (from after the VLED to 3 years), nor in period III (from baseline to 3 years) analyzed through a mixed linear model adjusting for age, gender, site, and baseline category of normal versus elevated fasting plasma glucose also adjusting for interaction with time (Fig. 2). A nonsignificant tendency towards lower levels of fibrinogen in the placebo group was present at 3 years $(P=0.06)$. A multiple linear regression for periods I-III also including weight change was made in order to check whether weight change was associated with changes in adiponectin, hs-CRP, and fibrinogen and also to check for whether the effects of weight change might depend upon treatment group. Two results emerged from this analysis: 1) weight change was significantly negatively associated with changes in adiponectin independent of treatment, gender, age, site, and fasting plasma glucose level during the study $(P<0.01)$. Changes in hs-CRP were significantly $(P<0.05)$ positively associated with changes in weight from baseline to 3 years (period III) but not during the VLED (period I; $P=0.06$ ) nor from the end of the VLED to 3 years (period II). Changes in fibrinogen are not associated with changes in weight during the study. 2) Treatment by itself adjusted for the mentioned factors was not significantly associated with changes in adiponectin, hs-CRP, and fibrinogen. As visualized in Fig. 2, changes in the various markers of inflammation did not differ between the two treatment groups during the study. These findings are in accordance with the results from the original cohort finding that no significant differences between metabolic changes in the two treatment groups were observed (19). In order to increase the power of the analysis, we pooled the results from the two treatment groups (orlistat and placebo) and further analysis of the effect of weight change was conducted on all subjects taken together.

Adiponectin increased significantly by $22 \%(-10$ to $+50 \%$ ) median (interquartile range; $P<0.05$ ) from baseline to the end of VLED (period I; Fig. 3). Six months after the VLED, adiponectin was still significantly 


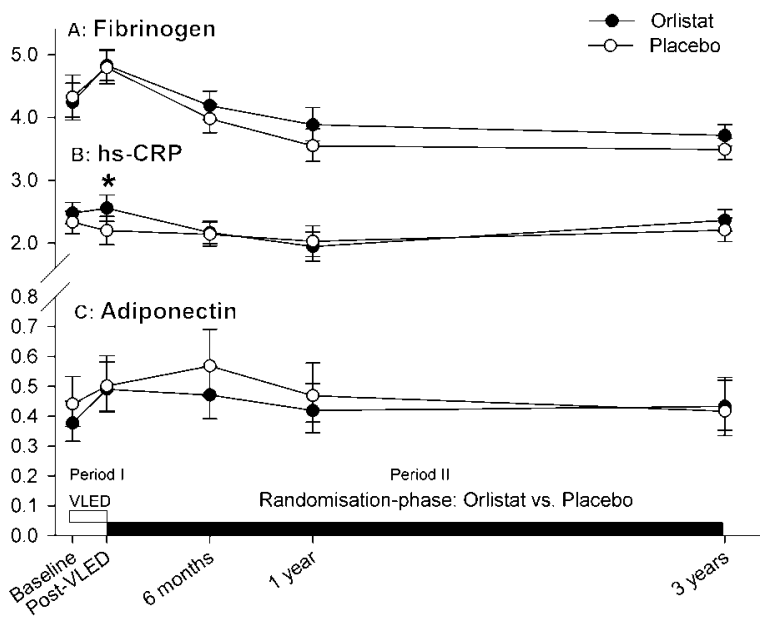

Figure 2 Adiponectin, hs-CRP and fibrinogen according to treatment groups. Values are A: fibrinogen (g/L), B: hs-CRP ( $\mu \mathrm{g} / \mathrm{ml})$. C: adiponectin $(\mu \mathrm{g} / \mathrm{ml})$. A and $\mathrm{C}$ represent geometric and $\mathrm{B}$ arithmetic means with $95 \% \mathrm{Cl}$ adjusted for gender, site, age, and normal versus elevated fasting plasma glucose according to treatment groups: orlistat (๑) or placebo (O). ${ }^{\star} P<0.05$ for differences between levels. No significant differences between the treatment groups regarding changes from baseline: periods I and III or from the end of the VLED: period II; $n=63-68$.

elevated by $24 \%$ ( 0 to $+55 \% ; P<0.05)$ compared with baseline levels. Thereafter, adiponectin decreased and at 3 years, no significant changes compared with baseline levels were detected in the whole group, despite a still significant body weight reduction $(\sim 7.5 \mathrm{~kg}$; Fig. 1$)$.

hs-CRP did not change significantly from baseline to the end of the VLED (period I) but was significantly reduced by $10 \%(+8$ to $-25 \%) 6$ months after the VLED $(P<0.05)$ and by $18 \%(-2$ to $-36 \%)$ after 1 year $(P<0.05)$. However, after 3 years, only a non-significant reduction was found compared with baseline (period III; Fig. 3).

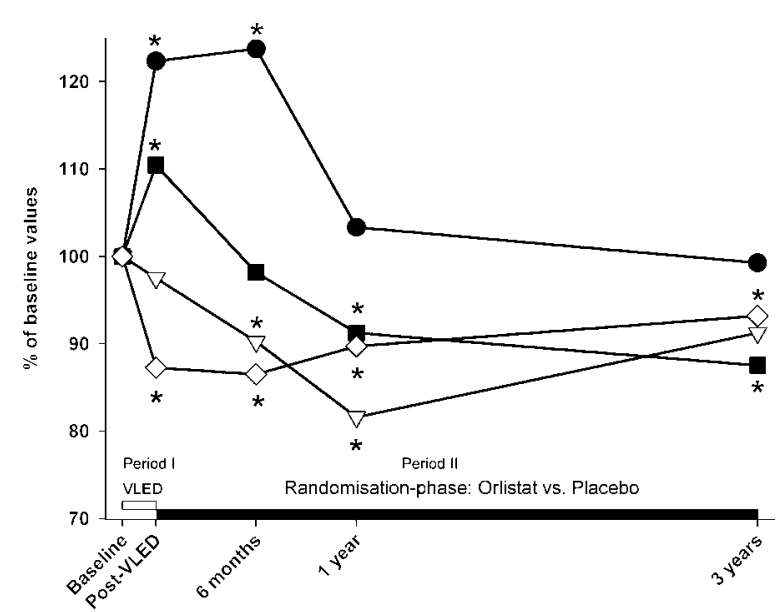

Figure 3 Weight-related changes in levels of inflammatory markers. Relative changes in adiponectin $(\bullet)$, hs-CRP $(\nabla)$, fibrinogen ( $(\mathbf{\square})$, and weight $(\diamond)$ during the study. Data are medians of relative changes compared with baseline values. $N=63-68$. ${ }^{*} P<0.05$ as compared with baseline.
Compared with baseline, fibrinogen increased significantly by $+10 \%(-1$ to $+27 \%)$ at the end of the VLED (period I; $P<0.05$ ) but was found to be significantly reduced by $-12 \%(-2$ to $-25 \%)$ after 3 years (period III; $P<0.05$; Fig. 3).

\section{Influence of the degree of weight loss on adiponectin, hs-CRP and fibrinogen}

In order to investigate the significance of the degree of weight loss upon changes in adiponectin, a linear mixed model was established for changes in adiponectin from baseline to 3 years (period III) adjusted for treatment, gender, site, and normal versus elevated fasting plasma glucose using age and weight change as covariates. This model confirmed the significant $(P<0.01)$ negative relationship between changes in adiponectin and changes in weight. The analysis showed that a weight loss of $10 \%$ resulted in a significant $11 \%$ increase in adiponectin with 95\% confidence intervals (0-23\%) after adjustment. This relationship is illustrated by the scatterplot in Fig. 4 showing unadjusted changes in weight and adiponectin with $95 \%$ confidence intervals of the predicted mean indicating that a weight loss of $\sim 10 \%$ or more is needed for a significant increase in adiponectin.

In order to further investigate these findings on unadjusted values, we divided the population into quartiles of relative weight loss from baseline to the end of the VLED (period I) and from baseline to 3 years (period III).

Adiponectin A VLED-induced weight loss larger than $12 \%$ corresponding to more than $13 \mathrm{~kg}$ weight loss resulted in significantly elevated levels of adiponectin of $+22.3 \%$ ( -10 to $+49.5 \%) P<0.01$ (data not shown),

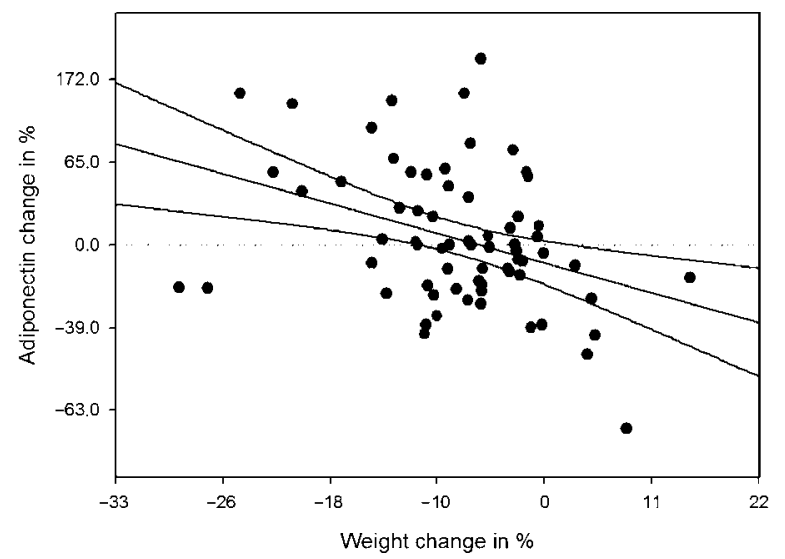

Figure 4 Scatterplot with relative changes in adiponectin (\%) from baseline to 3 years (period III) plotted by changes in weight (\%). Regression curve with $95 \%$ confidence interval of predicted mean together with reference line indicating baseline adiponectin levels ( $0 \%$ change). The values are unadjusted log (base e) differences transformed into relative changes explaining the non-linear scale; $n=68$. 
whereas weight losses below $12 \%$ only induced nonsignificant increases in adiponectin levels. From baseline to 3 years (period III), the relative increase in adiponectin (percentage of baseline values) was significantly higher $(P<0.01)$ when comparing subjects losing $11.2-28.9 \%$ of baseline weight (highest quartile) compared with subjects with a weight change of -2.5 to $+14.5 \%$ (lowest quartile). Moreover, only subjects from the highest weight loss quartile during period III (weight loss $>11 \%$ ) achieved a significantly increased adiponectin level $(P<0.05$; Fig. 5), when comparing changes from baseline to 3 years.

Hs-CRP Relative levels of hs-CRP did not differ significantly between the quartiles of relative weight change after the VLED (period I) and even the subjects from the highest weight loss quartile did not have a significant reduction of hs-CRP compared with baseline.
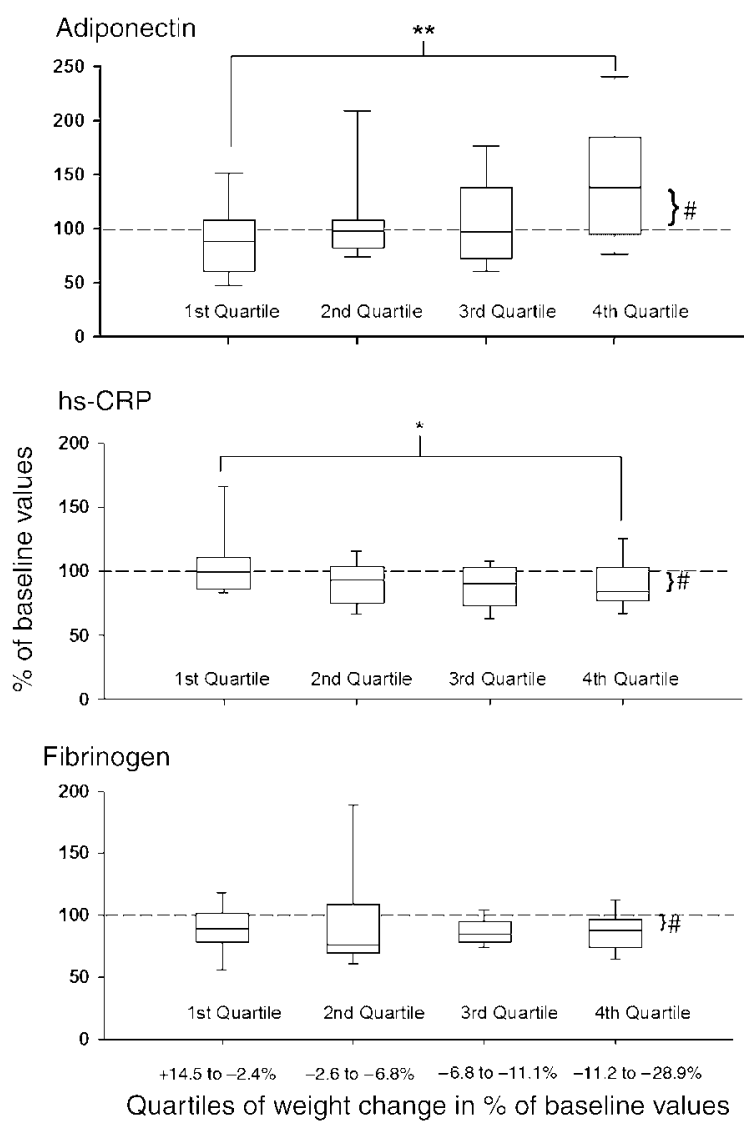

Figure 5 Effect of the level of weight loss on changes in inflammatory markers. The population was divided according to the degree of weight loss from baseline to 3 years. Relative levels of adiponectin, hs-CRP, and fibrinogen are shown according to quartiles of relative weight changes. Data are presented as box plots where the upper and lower lines of the boxes represent the 25th and 75th percentiles and the middle line represents the median value. Whiskers represent 5 th and 95th percentiles. $N=66-68$. ${ }^{\star} P<0.05$ and ${ }^{\star *} P<0.01$, comparison of first versus fourth weight change quartile. ${ }^{\#} P<0.05$, comparison of baseline levels with the fourth weight change quartile.
After 3 years, the decrease in hs-CRP was significantly larger in subjects from the highest versus the lowest quartile of relative weight change $(P<0.05$, Fig. 5). After 3 years, weight losses larger than $11 \%$ were also associated with a significantly lower hs-CRP level than baseline levels $(P<0.05$, Fig. 5). A significantly lower level of hs-CRP $(P=0.015)$ was also found in the subjects with the highest $(>10.8 \%)$ compared with those with the lowest relative waist circumference decrease (data not shown).

Fibrinogen Relative levels of fibrinogen did not differ significantly between quartiles of relative weight loss after the VLED (data not shown) nor after 3 years (Fig. 5)

Lipids, HbA1c and HOMA-IR The changes (\%) in lipids, HbAlc, and HOMA-IR according to the highest and lowest quartiles of weight change (\%) from baseline to 3 years (period III) are referred in Table 2. Subjects from the fourth quartile $(-11.2$ to $-28.9 \%$ weight loss) had significantly larger decreases in HOMA-IR $(P<0.001)$, HbA1c $(P<0.001)$, and triglycerides $(P<0.01)$ as well as larger increases in HDL cholesterol $(P<0.05)$ compared with subjects from the first quartile $(+14.5$ to $-2.4 \%)$, but no significant differences regarding changes in total and LDL cholesterol were detected.

\section{Bivariate correlations}

Changes in adiponectin after the VLED treatment (period I) correlated negatively with weight loss $(r=-0.27$, $P<0.05)$ and positively with changes in HDL cholesterol $(r=0.23, P=0.05)$ but was not correlated $(r=-0.03)$ with changes in HOMA-IR. After 3 years, changes in adiponectin were correlated with weight loss $(r=-0.33$, $P<0.01)$ and HDL cholesterol $(r=0.30, P<0.05)$ in a similar way as shown already after 8 weeks. The changes in adiponectin were also negatively correlated with changes in insulin resistance $(r=-0.24, P=0.05)$ after 3 years. But the changes in hs-CRP correlated positively both with weight loss $(r=0.24, P<0.05)$ and with HOMA $(r=0.25, P<0.05)$ after 3 years. Fibrinogen did not correlate to any variables in this study.

\section{Discussion}

A relatively large weight loss was induced by 8 weeks of VLED (period I) followed by a 3-year randomized phase (period II) with treatment with either orlistat or placebo during which the majority of the subjects regained weight. The treatment with orlistat did not result in a significantly larger overall weight loss compared with placebo when including the effect of the VLED (baseline to 3 years; period III). But orlistat treatment did diminish the weight gain from the end of the VLED to 
Table 2 Relative changes in metabolic parameters (\%) from baseline to 3 years according to weight loss quartiles (\%).

\begin{tabular}{|c|c|c|c|}
\hline & $\begin{array}{l}\text { First quartile of weight loss } \\
\text { (range: }+14.5 \text { to }-2.5 \% ; n=17) \\
\text { Mean }(95 \% \mathrm{Cl})\end{array}$ & $\begin{array}{l}\text { Fourth quartile of weight loss } \\
\text { (range: }-11.2 \text { to }-28.9 \% ; n=17) \\
\text { Mean }(95 \% \mathrm{Cl})\end{array}$ & $\begin{array}{l}\text { All subjects (range: }+14.5 \text { to } \\
-28.9 \% ; n=68) \\
\text { Mean }(95 \% \mathrm{Cl})\end{array}$ \\
\hline $\begin{array}{l}\Delta \text {-Weight } \\
\Delta-\text { Total cholesterol } \\
\Delta-\text { LDL cholesterol } \\
\Delta-\text { HDL cholesterol } \\
\Delta-\text { Triglyceride } \\
\Delta-\text { HbA1c } \\
\Delta-\text { HOMA-IR }\end{array}$ & $\begin{array}{l}+1.3 \%(+3.6 \text { to }-1.0) \\
-2.2 \%(+11.8 \text { to }-14.4) \\
-8.6 \%(+9.4 \text { to }-23.6) \\
-4.7 \%(+1.8 \text { to }-10.8) \\
20.6 \%(+45.6 \text { to }-0.1) \\
-8.1 \%(-5.9 \text { to }-10.3) \\
24.8 \%(+63.9 \text { to }-5.0)\end{array}$ & $\begin{aligned} &-17.4 \%(-14.3 \text { to }-20.3)^{\ddagger} \\
&-8.9 \%(-4.5 \text { to }-13.1) \\
&-9.2 \%(-3.2 \text { to }-14.9) \\
& 10.4 \%(24 \text { to }-1.7)^{\star} \\
&-32.7 \%(-11.8 \text { to }-48.7)^{\dagger} \\
&-17.7 \%(-13 \text { to }-22.2)^{\ddagger} \\
&-59 \%(-41.3 \text { to }-71.4)^{\ddagger}\end{aligned}$ & $\begin{array}{r}-7.8 \%(-5.8 \text { to }-9.7)^{\llbracket} \\
-7.5 \%(-2.9 \text { to }-11.8)^{\|} \\
-10.5 \%(-4.1 \text { to }-16.4)^{\|} \\
1.6 \%(6.1 \text { to }-2.7) \\
-12 \%(-1.3 \text { to }-21.5)^{\S} \\
-13.1 \%(-11.3 \text { to }-14.9)^{\Phi} \\
-29.9 \%(-17.3 \text { to }-40.5)^{\Phi}\end{array}$ \\
\hline
\end{tabular}

${ }^{\star} P<0.05,{ }^{\dagger} P<0.01$, and ${ }^{\ddagger} P<0.001$; fourth versus first quartile of weight loss (\%), ANOVA with adjustment for multiple comparisons (Dunnet). ${ }^{\S} P<0.05$ unadjusted, "P<0.01, and ${ }^{~} P<0.001$ for changes from baseline to 3 years (period III) in all subjects. Linear mixed model for repeated measurements with adjustment for multiple comparisons (Sidak). Values are geometric means with $95 \% \mathrm{Cl}$ of relative changes (transformed from log changes (base e)); $n=67-68$.

3 years (period II) (19). However, this difference was not associated with any significant differences between changes in the inflammatory markers in the two groups. Accordingly, the results presented are the changes of the combined orlistat and placebo group.

Interestingly, we found that a mean weight loss of $\sim 14 \mathrm{~kg}$ after 8 weeks of VLED resulted in a significant $22 \%$ increment in serum levels of the adipocyte-specific protein adiponectin. In contrast, a smaller weight loss (e.g. 5-7 kg) was unable to increase plasma adiponectin significantly neither in the short term ( 8 weeks) nor in the long term (3 years). We also found that relatively large weight losses (more than 10-11\%) were necessary in order to obtain a continuous significant increase in plasma adiponectin levels. These results may in part explain some of the discrepancy between the various studies reporting no association between weight losses in the range of $6.5-8 \%$ and adiponectin increase (24, 25) compared with significant increases in plasma adiponectin seen with a weight loss of $\sim 14-15 \%(16)$.

Adiponectin is of particular interest due to its inverse association with obesity and its stipulated insulinsensitizing effects (13). Moreover, adiponectin may play a central role for the low-grade inflammation milieu found in obesity - being inversely associated with IL-6 and TNF- $\alpha(16,26)$ - and through its ability to stimulate release of anti-inflammatory proteins such as IL-10 and IL-1 receptor antibodies (27). The visceral AT depot seems to be more proinflammatory than the s.c. as demonstrated by a higher release of proinflammatory adipokines (6), more macrophage infiltration $(7,28)$, and lower adiponectin expression (29). In crosssectional studies, the visceral AT depot is suggested to be an important determinant of circulating adiponectin levels, indicating that reduced adiponectin might link visceral obesity with the insulin resistance/metabolic syndrome (30). However, our data indicate that a substantial relative weight reduction over 3 years is needed for lasting increases in adiponectin levels.

The validated markers of chronic low-grade inflammation hs-CRP and fibrinogen were also affected by weight loss with a significant reduction in hs-CRP up to 12 months after the VLED with a non-significant tendency to remain reduced after 3 years. Parallel to our findings on adiponectin, we found that the longterm (3 years) weight loss needed to be larger than 10\% in order to induce a significant reduction in hs-CRP. Several other studies have also found that weight loss in obese patients is associated with reductions in hs-CRP $(26,31-33)$. Some of these studies have a shorter follow-up period and it may therefore be difficult to distinguish between the effects of the hypocaloric state induced by diet from the weight loss per se. The data presented in this study indicate that a relatively large net weight loss is needed in order to be associated with a stable reduction in hs-CRP levels up to 3 years. Plasma fibrinogen increased transiently after the VLED but was followed by a long-term significant reduction. The exact mechanism for the VLED-induced increase in fibrinogen remains unknown but it has been shown that starvation and intracellular mobilization of fatty acids may represent a stress to, for example, the hepatic metabolism resulting in increased generation of acute phase reactants (34). A similar explanation can be proposed for the lack of decrease in hs-CRP levels following the VLED in the present study. This tendency was also noted in a study where the weight loss was induced by gastric restrictive surgery also showing increased levels of acute phase reactants (35).

In the present study, we have not investigated whether weight loss was differently associated with the various molecular forms of adiponectin (high, medium, and low molecular weight) but a recent study (36) has shown that total adiponectin increased by $36 \%$ after weight loss and the various molecular forms of adiponectin all increased to a similar degree.

We found no additional effects of orlistat treatment on plasma levels of adiponectin, hs-CRP, and fibrinogen, which is in accordance with our recent study where no extra effects of orlistat were seen on the metabolic profile (19). This fact may be related to the pronounced metabolic effects induced by the VLED phase (inducing a mean weight loss of $14 \mathrm{~kg}$ ) that may mask the possibly more discrete effects of orlistat (23). Generalization of these findings, considering the possible intervention effect of the regime described, must take into 
consideration that they are based on a selected population with the capability of losing at least $5 \%$ of their body mass during 8 weeks of VLED and complete 3 years of participation in a formalized study. But even though we did not find any additive effect of orlistat treatment following 8 weeks of VLED, this does not preclude possible effects of orlistat in other studies with different types of adjuvant weight loss support. In fact, other studies have found that orlistat treatment may induce independent reductions in the levels of triglycerides, hs-CRP, TNF- $\alpha$, insulin-like growth factor-I and isoprostane $(37,38)$, or improvements in insulin sensitivity and free fatty acids at equivalent weight losses compared with placebo (39). However, these studies lasting 6-12 months may explain why decreasing longterm effects induced by lessened compliance or adaption to the drug might not be seen.

It is well known that weight losses in the range of $2.5-5 \%$ are able to improve factors of the metabolic syndrome such as insulin sensitivity (40). Although several studies have been published concerning weight loss and subsequent changes in inflammatory markers $(15,31,41)$, it still remains unknown what the magnitude of weight loss should be in order to obtain long-term improvements in these inflammatory markers. But our present study indicates that higher weight losses are necessary to affect the inflammatory markers than are needed to affect insulin sensitivity.

In conclusion, we found a dose-effect relationship between the degree of weight loss and the improvement in plasma levels of adiponectin and hs-CRP. A net weight loss larger than $10 \%$ of the initial body weight seemed necessary for long-term combined improvement of adiponectin, hs-CRP and fibrinogen levels.

\section{Acknowledgements}

The study was sponsored by Roche and Aarhus University. The SMOMS trial from which these data originated is registered as study M37017 at http:// www.roche-trials.com/patient/trialresults/drugplst_ Xenical_orlistat.html.

\section{References}

1 Chan JC, Cheung JC, Stehouwer CD, Emeis JJ, Tong PC, Ko GT \& Yudkin JS. The central roles of obesity-associated dyslipidaemia, endothelial activation and cytokines in the metabolic syndrome - an analysis by structural equation modelling. International Journal of Obesity and Related Metabolic Disorders 200226 994-1008.

2 Third Report of the National Cholesterol Education Program (NCEP). Expert panel on detection, evaluation, and treatment of high blood cholesterol in adults (Adult Treatment Panel III) Final Report. Circulation 2002106 3143-3421.

3 Yusuf S, Hawken S, Ounpuu S, Bautista L, Franzosi MG, Commerford P, Lang CC, Rumboldt Z, Onen CL, Lisheng L, Tanomsup S, Wangai P Jr, Razak F, Sharma AM \& Anand SS.
Obesity and the risk of myocardial infarction in 27000 participants from 52 countries: a case-control study. Lancet 2005366 1640-1649.

4 Weyer C, Funahashi T, Tanaka S, Hotta K, Matsuzawa Y, Pratley RE \& Tataranni PA. Hypoadiponectinemia in obesity and type 2 diabetes: close association with insulin resistance and hyperinsulinemia. Journal of Clinical Endocrinology and Metabolism 200186 1930-1935.

5 Trayhurn P \& Wood IS. Adipokines: inflammation and the pleiotropic role of white adipose tissue. British Journal of Nutrition $200492347-355$.

6 Fain JN, Madan AK, Hiler ML, Cheema P \& Bahouth SW. Comparison of the release of adipokines by adipose tissue, adipose tissue matrix, and adipocytes from visceral and subcutaneous abdominal adipose tissues of obese humans. Endocrinology 2004 145 2273-2282.

7 Bruun JM, Lihn AS, Pedersen SB \& Richelsen B. Monocyte chemoattractant protein-1 release is higher in visceral than subcutaneous human adipose tissue (AT): implication of macrophages resident in the AT. Journal of Clinical Endocrinology and Metabolism $2005902282-2289$.

8 Woo KS, Chook P, Yu CW, Sung RY, Qiao M, Leung SS, Lam CW, Metreweli C \& Celermajer DS. Overweight in children is associated with arterial endothelial dysfunction and intima-media thickening. International Journal of Obesity and Related Metabolic Disorders 200428 852-857.

9 Yudkin JS, Kumari M, Humphries SE \& Mohamed-Ali V. Inflammation, obesity, stress and coronary heart disease: is interleukin-6 the link? Atherosclerosis 2000148 209-214.

10 Caballero AE. Endothelial dysfunction in obesity and insulin resistance: a road to diabetes and heart disease. Obesity Research 200311 1278-1289.

11 Ouchi N, Kihara S, Arita Y, Okamoto Y, Maeda K, Kuriyama H, Hotta K, Nishida M, Takahashi M, Muraguchi M, Ohmoto Y, Nakamura T, Yamashita S, Funahashi T \& Matsuzawa Y. Adiponectin, an adipocyte-derived plasma protein, inhibits endothelial NF- $\kappa \mathrm{B}$ signaling through a cAMP-dependent pathway. Circulation 2000102 1296-1301.

12 Okamoto Y, Kihara S, Ouchi N, Nishida M, Arita Y, Kumada M, Ohashi K, Sakai N, Shimomura I, Kobayashi H, Terasaka N, Inaba T, Funahashi $\mathrm{T}$ \& Matsuzawa Y. Adiponectin reduces atherosclerosis in apolipoprotein E-deficient mice. Circulation $20021062767-2770$.

13 Lihn AS, Pedersen SB \& Richelsen B. Adiponectin: action, regulation and association to insulin sensitivity. Obesity Reviews 20056 13-21.

14 Yang WS, Lee WJ, Funahashi T, Tanaka S, Matsuzawa Y, Chao CL, Chen CL, Tai TY \& Chuang LM. Weight reduction increases plasma levels of an adipose-derived anti-inflammatory protein, Adiponectin. Journal of Clinical Endocrinology and Metabolism 200186 3815-3819.

15 Esposito K, Pontillo A, Di Palo C, Giugliano G, Masella M, Marfella R \& Giugliano D. Effect of weight loss and lifestyle changes on vascular inflammatory markers in obese women: a randomized trial. Journal of the American Medical Association 2003289 1799-1804.

16 Bruun JM, Lihn AS, Verdich C, Pedersen SB, Toubro S, Astrup A \& Richelsen B. Regulation of adiponectin by adipose tissue-derived cytokines: in vivo and in vitro investigations in humans. American Journal of Physiology. Endocrinology and Metabolism 2003285 E527-E533.

17 Xydakis AM, Case CC, Jones PH, Hoogeveen RC, Liu MY, Smith EO, Nelson KW \& Ballantyne CM. Adiponectin, inflammation, and the expression of the metabolic syndrome in obese individuals: the impact of rapid weight loss through caloric restriction. Journal of Clinical Endocrinology and Metabolism 200489 2697-2703.

18 Abbasi F, Chang SA, Chu JW, Ciaraldi TP, Lamendola C, McLaughlin T, Reaven GM \& Reaven PD. Improvements in insulin resistance with weight loss, in contrast to rosiglitazone, are not 
associated with changes in plasma adiponectin or adiponectin multimeric complexes. American Journal of Physiology. Regulatory, Integrative and Comparative Physiology 2006290 R139-R144.

19 Richelsen B, Tonstad S, Rossner S, Toubro S, Niskanen L, Madsbad S, Mustajoki P \& Rissanen A. Effect of orlistat on weight regain and cardiovascular risk factors following a very-low-energy diet in abdominally obese patients: A 3-year randomized, placebocontrolled study. Diabetes Care 200730 27-32.

20 Laukkanen R, Oja P, Pasanen M \& Vuori I. Validity of a two kilometre walking test for estimating maximal aerobic power in overweight adults. International Journal of Obesity and Related Metabolic Disorders 199216 263-268.

21 Clauss A. Rapid physiological coagulation method in determination of fibrinogen. Acta Haematologica 195717 237-246.

22 Skogstrand K, Thorsen P, Norgaard-Pedersen B, Schendel DE, Sorensen LC \& Hougaard DM. Simultaneous measurement of 25 inflammatory markers and neurotrophins in neonatal dried blood spots by immunoassay with xMAP technology. Clinical Chemistry 200551 1854-1866.

23 Martins TB, Pasi BM, Litwin CM \& Hill HR. Heterophile antibody interference in a multiplexed fluorescent microsphere immunoassay for quantitation of cytokines in human serum. Clinical and Vaccine Immunology 200411 325-329.

24 Ryan AS, Nicklas BJ, Berman DM \& Elahi D. Adiponectin levels do not change with moderate dietary induced weight loss and exercise in obese postmenopausal women. International Journal of Obesity and Related Metabolic Disorders 200327 1066-1071.

25 Dvorakova-Lorenzova A, Suchanek P, Havel PJ, Stavek P, Karasova L, Valenta Z, Tintera J \& Poledne R. The decrease in C-reactive protein concentration after diet and physical activity induced weight reduction is associated with changes in plasma lipids, but not interleukin-6 or adiponectin. Metabolism 200655 359-365.

26 Engeli S, Feldpausch M, Gorzelniak K, Hartwig F, Heintze U, Janke J, Mohlig M, Pfeiffer AFH, Luft FC \& Sharma AM. Association between adiponectin and mediators of inflammation in obese women. Diabetes 200352 942-947.

27 Wolf AM, Wolf D, Rumpold H, Enrich B \& Tilg H. Adiponectin induces the anti-inflammatory cytokines IL-10 and IL-1RA in human leukocytes. Biochemical and Biophysical Research Communications 2004323 630-635.

28 Cancello R, Tordjman J, Poitou C, Guilhem G, Bouillot JL, Hugol D, Coussieu C, Basdevant A, Hen AB, Bedossa P, Guerre-Millo M \& Clement K. Increased infiltration of macrophages in omental adipose tissue is associated with marked hepatic lesions in morbid human obesity. Diabetes 200655 1554-1561.

29 Lihn AS, Bruun JM, He G, Pedersen SB, Jensen PF \& Richelsen B. Lower expression of adiponectin mRNA in visceral adipose tissue in lean and obese subjects. Molecular and Cellular Endocrinology 2004219 9-15.

30 Mori Y, Hoshino K, Yokota K, Itoh Y \& Tajima N. Differences in the pathology of the metabolic syndrome with or without visceral fat accumulation: a study in pre-diabetic Japanese middle-aged men. Endocrine 200629 149-153.
31 The Diabetes Prevention Program Research Group. Intensive lifestyle intervention or metformin on inflammation and coagulation in participants with impaired glucose tolerance. Diabetes $2005 \mathbf{5 4} 1566-1572$.

32 Heilbronn LK \& Clifton PM. C-reactive protein and coronary artery disease: influence of obesity, caloric restriction and weight loss. Journal of Nutritional Biochemistry 200213 316-321.

33 Clifton PM, Keogh JB, Foster PR \& Noakes M. Effect of weight loss on inflammatory and endothelial markers and FMD using two low-fat diets. International Journal of Obesity 200529 1445-1451.

34 Drenick EJ, Simmons F \& Murphy JF. Effect on hepatic morphology of treatment of obesity by fasting, reducing diets and small-bowel bypass. New England Journal of Medicine 1970282 829-834.

35 van Dielen FMH, Buurman WA, Hadfoune M, Nijhuis J \& Greve JW. Macrophage inhibitory factor, plasminogen activator inhibitor-1, other acute phase proteins, and inflammatory mediators normalize as a result of weight loss in morbidly obese subjects treated with gastric restrictive surgery. Journal of Clinical Endocrinology and Metabolism $2004894062-4068$.

36 Polak J, Kovacova Z, Jacek M, Klimcakova E, Kovacikova M, Vitkova M, Kuda O, Sebela M, Samcova E \& Stich V. An increase in plasma adiponectin multimeric complexes follows hypocaloric diet-induced weight loss in obese and overweight premenopausal women. Clinical Science $2007112557-565$.

37 Bougoulia M, Triantos A \& Koliakos G. Effect of weight loss with or without orlistat treatment on adipocytokines, inflammation, and oxidative markers in obese women. Hormones 20065 259-269.

38 Samuelsson L, Gottsater A \& Lindgarde F. Decreasing levels of tumour necrosis factor alpha and interleukin 6 during lowering of body mass index with orlistat or placebo in obese subjects with cardiovascular risk factors. Diabetes, Obesity and Metabolism 2003 5 195-201.

39 Kelley DE, Kuller LH, McKolanis TM, Harper P, Mancino J \& Kalhan S. Effects of moderate weight loss and orlistat on insulin resistance, regional adiposity, and fatty acids in type 2 diabetes. Diabetes Care 200427 33-40.

40 Lofgren IE, Herron KL, West KL, Zern TL, Brownbill RA, Ilich JZ, Koo SI \& Fernandez ML. Weight loss favorably modifies anthropometrics and reverses the metabolic syndrome in premenopausal women. Journal of the American College of Nutrition 200524 486-493.

41 Bruun JM, Verdich C, Toubro S, Astrup A \& Richelsen B. Association between measures of insulin sensitivity and circulating levels of interleukin-8, interleukin-6 and tumor necrosis factor-alpha. Effect of weight loss in obese men. European Journal of Endocrinology $2003 \mathbf{1 4 8} 535-542$.

Received 14 October 2007

Accepted 17 November 2007 